\title{
Identification of contact dynamics parameters for stiff robotic payloads
}

\author{
Diederik Verscheure*, Inna Sharf ${ }^{\dagger}$, Herman Bruyninckx*, Jan Swevers*, and Joris De Schutter*
}

\begin{abstract}
This paper investigates and demonstrates the feasibility of identifying contact dynamics parameters for stiff robotic payloads using a robotic system. The contact dynamics model for stiff payloads is motivated and theoretical parameter values and bounds are provided. Then, the effect of non-idealities such as surface roughness and plastic deformation on the theoretical values is demonstrated. A row-wise scaled total least squares parameter estimation algorithm is proposed and applied to experimental data measured using the Special Purpose Dexterous Manipulator (SPDM) Task Verification Facility (STVF) manipulator at the Canadian Space Agency (CSA). The experimental results are compared to a separate set of experiments with a material testing machine as well as finite element modeling results. Finally, the experimental findings are generalized by providing guidelines for the maximum identifiable payload stiffness as a function of the position resolution, the maximum exertable force and the structural stiffness of the robotic system.
\end{abstract}

\section{INTRODUCTION}

$\mathbf{R}$ OBOTS are increasingly used to perform complex tasks, which often involve contact with the environment. Constrained robotic tasks remain challenging, however, because the dynamic behavior of the environment affects the accuracy and stability of the low-level controllers. Therefore, computer simulations [1]-[3] play an essential role in the design and validation of constrained robotic operations and are especially important for tasks where prior testing is difficult, if not impossible, such as space operations [4], [5]. The fidelity of these simulations, however, depends on the use of reliable models of the environment dynamics, which in general consist of the structural dynamics of the environment and the dynamics of the contact between the robotic system and the environment. The reliability of these models, in turn, depends on accurate specification of model parameters. Of particular difficulty is the specification of contact dynamics parameters, which can vary greatly with the contact geometry, surface quality and other characteristics of the contacting objects. Only in a limited number of cases, analytical results, such as Hertz's theory [6], are available to calculate these parameters. More often, however, contact dynamics parameters need to be determined experimentally. To avoid time-consuming and costly experiments with dedicated hardware, identification of contact dynamics parameters from typical robotic operations using standard robotic hardware is a topic of on-going research.

\footnotetext{
*D. Verscheure, H. Bruyninckx, J. Swevers and J. De Schutter are with the Division PMA, Department of Mechanical Engineering, Katholieke Universiteit Leuven, Belgium.

$\dagger_{\mathrm{I}}$. Sharf is with the Department of Mechanical Engineering, McGill University, Montreal, Quebec, Canada, e-mail: inna.sharf@mcgill.ca.

Manuscript received January xx, 2008; revised January xx, 2008.
}

Initial efforts in on-line identification of environment $d y$ namics were motivated by the need to improve contact stability and force tracking in the context of force and impedance control [7]-[15]. In this context, the environment dynamics are approximated by a linear spring, spring-damper or massspring-damper model and the model parameters are estimated using adaptive control methods [8], [10], [12], (extended) Kalman filtering [9], [13], [15], recursive least squares [11] or active observers [14]. Although often negligible, the identified dynamics also contain the structural dynamics of the robotic system itself.

Unlike these control-inspired approaches, where only a reasonable estimate of the environment dynamics is needed, recent work has increasingly focused on accurate identification of environment and more specifically contact dynamics, for purposes such as computer simulations or detection and perception [16], [17]. As a result, a number of performance investigations [18]-[20] have been carried out and various onand off-line algorithms have been developed and refined [18][24]. Furthermore, in certain cases, the dynamics of the robotic system are no longer neglected [19], [20]. In [18], [23], the performance of some of the early on-line methods [10]-[12] is compared, while these methods are refined for off-line processing and compared in [21]. In [21], [22], a time-domain methodology is developed to identify contact dynamics parameters for complex multi-point contact scenarios. In addition to the three on-line methods in [10]-[12], a frequency-domain method is presented in [18], [23], while a number of other frequency-domain methods are also discussed in [24]. In [19], [20], several on- and off-line time- and frequency-domain methods are compared for the estimation of contact dynamics parameters for stiff robotic payloads.

In the pursuit of accurate identification of environment and contact dynamics, attention has also turned toward estimation of parameters of nonlinear contact dynamics models. In [25], a fully empirical approach is used to determine the parameters of a nonlinear contact model for a space robotic insertion task. In [26], [27], an alternating recursive least squares (ARLS) method is used to identify the parameters of the nonlinear Hunt-Crossley model [28]-[30]. A multi-pass version of the ARLS method in [26], [27] which exhibits an improved convergence for the estimation of stiff contact dynamics is presented in [31].

In line with the objectives of this recent work, this paper is concerned with the estimation of contact dynamics parameters for stiff robotic payloads. Unlike the control-inspired approaches, our goal is to identify the contact dynamics of the payloads, excluding the structural dynamics of the 
robotic system and its worksite. Because of the nonlinearity of the contact dynamics, the small deformations and relatively high interaction forces involved, and the fallacy of the rigidbody approximation of the robotic system for stiff contact interactions, this topic is particularly challenging.

While expanding upon the work in [19], [20], [31], the perspective of this paper is considerably different and more fundamental in nature than [19], [20], [31], where the focus is mainly algorithmic. Specifically, this paper investigates and demonstrates the feasibility of estimating the contact dynamics parameters for stiff robotic payloads using robotic hardware, and identifies the impediments which render this a difficult task. The feasibility is investigated by means of robotic experiments and results are compared to an additional set of experiments as well as finite element modeling results. Based on the knowledge acquired in the identification process, feasibility guidelines are formulated that generalize the findings in this paper to other robotic systems and payloads.

The outline of this paper is as follows. Section II discusses the modeling of nonlinear contact dynamics. The choice of a Hertz-like nonlinear contact model for stiff payloads is motivated in Section II-A, while the effects of non-idealities such as surface roughness and plastic deformation are discussed in Sections II-B and II-C. Section III presents a row-wise scaled total least squares algorithm for both batch and recursive estimation of the contact dynamics parameters. Section IV discusses the experimental robotic facility, presents the experimental results and applies the parameter estimation algorithm to a number of different stiff payloads. Section V compares the robotic experiments with a separate set of experiments carried out using an Instron material testing machine and with finite element modeling results. In Section VI, we point out a number of error sources which influence the results and finally, in Section VII, we propose guidelines for the feasibility of contact dynamics parameter estimation for stiff robotic payloads. Section VIII formulates conclusions and points to future work.

\section{Modeling OF CONTACT DYNAMICS}

\section{A. Modeling of smooth elastic contact}

This section discusses the modeling of stiff single point contact scenarios. While linear models are widely and succesfully used in engineering practice, contact dynamics are intrinsically nonlinear due to the dependency of the contact area on the load [32]. The nonlinear force-deformation law by Hertz [6] is undoubtedly one of the most well-known results in modeling of elastic contacts. A similar power law is often adopted for more general geometries [28], [30], [33], without any motivation. In the following, we demonstrate the asymptotic validity, that is, for small deformations, of this Hertz-like power law and provide theoretical values and bounds for the parameters based on the local geometry of the contacting objects, following an extension of Hertz's theory [34], [35] for axisymmetric elastic bodies.

In [34], [35], a relation is derived between the radius $a$ of the contact area and the normal force, for two non-conforming contacting axisymmetric elastic bodies, where the distance $z$ in the undeformed state between the two bodies measured in the direction of the axis of symmetry, is described by a polynomial function of the distance $r$ to the axis of symmetry, as

$$
z=f(r)=\sum_{i=m}^{M} b_{i} r^{i},
$$

where $m>0$. When the radius $a$ of the contact area is small, or when $m=M$, such that $f(r)$ can be approximated as $f(r) \approx b_{m} r^{m}$ for $r \leq a$, it can be shown that the normal force $F$ follows a power law as a function of the deformation $\delta$, such that for small $\delta$

$$
F \approx K \delta^{n}
$$

where the parameters $K$ and $n$ are given by

$$
\begin{aligned}
K & =\sqrt{\pi} \frac{\Gamma\left(\frac{2+m}{2}\right)}{\Gamma\left(\frac{3+m}{2}\right)} E_{\text {eq }}\left(\frac{1}{b_{m}}\right)^{(1 / m)}, \\
E_{\text {eq }} & =\left(\frac{1-\nu_{1}^{2}}{E_{1}}+\frac{1-\nu_{2}^{2}}{E_{2}}\right)^{-1} \\
n & =\frac{m+1}{m}
\end{aligned}
$$

and where $E_{1}, E_{2}$ are the Young's moduli and $\nu_{1}, \nu_{2}$ the Poisson coefficients of the materials of the two contacting bodies. For example, for contact between two spheres with radii $R_{1}$ and $R_{2}$,

$$
b_{m}=\frac{1}{R_{\mathrm{eq}}}=\left(\frac{1}{R_{1}}+\frac{1}{R_{2}}\right) \text { and } m=2,
$$

and hence

$$
K=\frac{4}{3} E_{\text {eq }} \sqrt{R_{\text {eq }}} \text { and } n=\frac{3}{2},
$$

yielding Hertz's well-known result [6]. Similarly, the results for contact between a cone and a halfspace [32], and contact between a flat punch ${ }^{1}$ and a halfspace [32] can be obtained.

From (5), it follows that $1 \leq n \leq 2$ and hence, in all cases except the special case where $n=1$, a linear approximation of the force-deformation relation around $\delta=0$ does not exist, thereby illustrating the premise that contact dynamics are intrinsically nonlinear.

Equation (2) is exact only if $M=m$, but for more general geometries where $M>m$, the power law (2) is only an approximation for small deformations. Since the deformations are inherently small for stiff payloads, model (2) is a suitable choice for stiff axisymmetric payloads. While the power law model is not proven to be valid for non-axisymmetric objects, it is also applied to non-axisymmetric payloads. Analytical results for non-axisymmetric bodies, however, which are available only in a few cases [32], also support the validity of the power law model.

\footnotetext{
${ }^{1}$ Contact between a flat punch with radius $R$ and a halfspace, requires the unabbreviated approach as in [34], [35] and yields $K=2 R E_{\text {eq }}$ and $n=1$.
} 


\section{B. Modeling of contact between rough surfaces}

For stiff payloads, where the total deformation may be only a small multiple of the surface roughness, the contact area does not consist of a continuous contact zone, but rather of contact between individual asperities. Consequently, surface roughness can have an important effect on the force-deformation relation and the theoretical parameter values.

In [36], a compact description of contact between spheres with rough surfaces is presented in terms of dimensionless quantities. Since we are concerned with a global description of the contact dynamics in terms of the total force $F$ and the total deformation $\delta$, the results of [36] are briefly summarized from this perspective. Referring to equation (7), for ideal contact between spheres, the Hertzian deformation $\delta_{H}$ is given by

$$
\delta_{H}=\left(\frac{F}{K}\right)^{(1 / n)}=\left(\frac{3 F}{4 E_{\mathrm{eq}} \sqrt{R_{\mathrm{eq}}}}\right)^{(2 / 3)} .
$$

In contrast, for non-ideal contact of rough spheres [36], the deformation $\delta$ additionally depends on two parameters $\alpha$ and $\kappa$ which are defined as

$$
\alpha=\frac{\sigma_{\mathrm{eq}}}{\delta_{H}} \text { and } \kappa=\frac{E_{\mathrm{eq}}}{H_{\mathrm{mic}}} \sqrt{\frac{R_{\mathrm{eq}}}{\sigma_{\mathrm{eq}}}},
$$

where $\sigma_{\text {eq }}=\sqrt{\sigma_{1}^{2}+\sigma_{2}^{2}}$ is the equivalent roughness ${ }^{2}$ and $H_{\text {mic }}$ is the effective microhardness ${ }^{3}$. The deformation $\delta$ is then calculated as [36]

$$
\delta=\delta_{H}\left(0.5\left(a_{L}^{\prime}\right)^{2}+\frac{8 p_{0}^{\prime} a_{L}^{\prime}}{\pi^{2}\left(4.79-3.17\left(p_{0}^{\prime}\right)^{3.13}\right)}\right),
$$

as a function of the dimensionless maximum contact pressure $p_{0}^{\prime}$ and the relative contact radius $a_{L}^{\prime}$, given by

$$
\begin{aligned}
& p_{0}^{\prime}=\frac{1}{1+1.22 \alpha \kappa^{-0.16}}, \\
& a_{L}^{\prime}=\left\{\begin{aligned}
1.605 / \sqrt{p_{0}^{\prime}}, & \text { if } 0.01 \leq p_{0}^{\prime} \leq 0.47, \\
3.51-2.51 p_{0}^{\prime}, & \text { if } 0.47 \leq p_{0}^{\prime} \leq 1.0 .
\end{aligned}\right.
\end{aligned}
$$

As the equivalent roughness $\sigma_{\text {eq }}$ increases, both $\hat{K}$ and $\hat{n}$ of the best fitting power law increase significantly, resulting in a slower increase of the force for small deformations than would be expected from Hertz's theory and causing $n \geq 2$ to be possible in practice.

Moreover, the non-zero roughness causes an important deviation from the power law relation. For deformations significantly larger than $\sigma_{\text {eq }}, \alpha$ approaches 0 and $\delta$ approaches $\delta_{H}$, such that the force-deformation relations for different surface roughnesses asymptotically coincide. Consequently, for a nonzero roughness, no power law exists which is able to describe both the slower force onset for small deformations and the same asymptotic behavior for large deformations.

The effect of surface roughness is important if the surface roughness $\sigma_{\text {eq }}$ is larger than a few percent of the Hertzian or elastic deformation $\delta_{H}$, or in other words if $\alpha \geq 0.05$ [32].

\footnotetext{
${ }^{2}$ For a Gaussian surface [32], [36], the surface roughness $\sigma$ is defined as $\sqrt{\frac{\pi}{2}} R_{a}$ [32], [36], where $R_{a}$ is the well-known average roughness height.

${ }^{3}$ The effective microhardness $H_{\text {mic }}$ characterizes the effective indentation resistance against plastic deformation and is obtained using Vickers-like hardness tests on a microscopic scale.
}

\section{Modeling of elastic-plastic contact}

Even for moderate contact forces, local contact stresses may be very high, such that plastic deformation may occur. Plastic deformation causes not only deviations from the theoretical force-deformation relation [37], [38], but also time-varying behavior due to hardening effects [38], [39].

For contact between a sphere and a halfspace, the force $F_{c}$ and the deformation $\delta_{c}$, marking the transition from elastic to elastic-plastic behavior are given by [37]

$$
F_{c}=\frac{(\pi P H)^{3} R_{\mathrm{eq}}^{2}}{6 E_{\mathrm{eq}}^{2}} \text { and } \delta_{c}=\left(\frac{\pi P H}{2 E_{\mathrm{eq}}}\right)^{2} R_{\mathrm{eq}}
$$

where $P=0.454+0.41 \nu, H=2.8 Y$ and where $Y$ and $\nu$ are the yield stress and Poisson coefficient of the softest material in contact. In [37], [38], based on finite element modeling, the force-deformation relation accounting for elastic-plastic behavior, during the first loading, is given by

$$
F=\left\{\begin{array}{cl}
K \delta^{n}, & \text { if } \frac{\delta}{\delta_{c}} \leq 1 \\
1.03 F_{c}\left(\frac{\delta}{\delta_{c}}\right)^{1.425}, & \text { if } 1<\frac{\delta}{\delta_{c}} \leq 6 \\
1.40 F_{c}\left(\frac{\delta}{\delta_{c}}\right)^{1.263}, & \text { if } 6<\frac{\delta}{\delta_{c}} \leq 110
\end{array}\right.
$$

where $K$ and $n$ are given by equation (7). Consequently, $\hat{K}$ and $\hat{n}$ of the best fitting power law change from those given by equation (7) to $1.40 F_{c} / \delta_{c}^{1.263}$ and 1.263 respectively, depending on whether the force ranges from 0 to $F \leq F_{c}$, or from 0 to $F \gg F_{c}$. Rewriting equation (14) in a similar form as equation (10) yields

$$
\delta=\left\{\begin{array}{cl}
\delta_{H}, & \text { if } \frac{F}{F_{c}} \leq 1, \\
\delta_{H}\left(\frac{\delta_{c}\left(F^{(n / 1.425-1)} K\right)^{(1 / n)}}{\left(1.03 F_{c}\right)^{(1 / 1.425)}}\right), & \text { if } 1<\frac{F}{F_{c}} \leq 13.2, \\
\delta_{H}\left(\frac{\delta_{c}\left(F^{(n / 1.263-1)} K\right)^{(1 / n)}}{\left(1.40 F_{c}\right)^{(1 / 1.263)}}\right), & \text { if } 13.2<\frac{F}{F_{c}} \leq 530.2 .
\end{array}\right.
$$

While affecting the parameter values, elastic-plastic behavior also causes deviations from the power law model, as equation (15) consists of piecewise continuous power law segments, such that no single power law is able to describe the complete force-deformation relation.

Furthermore, equation (15) is unable to describe hardening effects which occur as a consequence of multiple loadingunloading cycles. The development and experimental validation of compact models which are able to describe these hardening effects, is still a topic of ongoing research [38], [39]. Of relevance to this paper is the result in [39], where it is found by means of finite element modeling, that if $F \gg F_{c}$ during the first few loading-unloading cycles, fully elastic steady state behavior occurs for subsequent loading-unloading cycles.

\section{CONTACT DyNAMiCs PARAMETER ESTIMATION}

The contact dynamics parameter estimation problem tackled in this paper, consists of estimating $K$ and $n$ of the power law model (2), from a series of measurements $\left(\hat{\delta}_{k}, \hat{F}_{k}\right)^{T}$

$$
\begin{aligned}
& \hat{\delta}_{k}=\delta_{k}+\Delta \delta_{k}, \\
& \hat{F}_{k}=F_{k}+\Delta F_{k},
\end{aligned}
$$


which are corrupted with noise terms $\Delta \delta_{k}$ and $\Delta F_{k}$ that are approximately zero-mean, serially and mutually independent and homoskedastic ${ }^{4}$ with known variances $\left(\sigma^{\delta}\right)^{2}$ and $\left(\sigma^{F}\right)^{2}$ respectively. The parameters $(\hat{K}, \hat{n})^{T}$ which best explain the measurements, can be obtained by minimizing the normalized mean squared error (NMSE) as

$$
\min _{K, n, \delta_{k}, F_{k}} \frac{1}{L} \sum_{k=0}^{L-1}\left[\frac{\left(\delta_{k}-\hat{\delta}_{k}\right)^{2}}{\left(\sigma^{\delta}\right)^{2}}+\frac{\left(F_{k}-\hat{F}_{k}\right)^{2}}{\left(\sigma^{F}\right)^{2}}\right],
$$

subject to $F_{k}=K \delta_{k}^{n}$, for $k=0 \ldots L-1$.

Unfortunately, problem (18)-(19) is very difficult to solve because of the nonlinearity of model constraint (19). Therefore, a relaxed approach similar to that in [27], [31] is adopted instead. Specifically, by applying a logarithmic transformation to equation (2) and a reparameterization $l=\ln (K)$,

$$
\ln \left(F_{k}\right)=\ln (K)+n \ln \left(\delta_{k}\right)=l+n \ln \left(\delta_{k}\right),
$$

model (20) becomes linear in the parameters $(l, n)^{T}$. Subsequently, by relaxing the noise assumptions and assuming $\ln \left(\hat{\delta}_{k}\right)$ and $\ln \left(\hat{F}_{k}\right)$ to be pseudo-measurements of $\ln \left(\delta_{k}\right)$ and $\ln \left(F_{k}\right)$ which are corrupted with zero-mean noise, least squares (LS) techniques can be used to estimate the parameters. Specifically, this paper adopts a row-wise scaled total least squares (RS-TLS) [40] approach, which is motivated by the following two considerations.

First, unlike [27], where relatively soft environments are considered, the deformation pseudo-measurements $\ln \left(\hat{\delta}_{k}\right)$ cannot be considered to be noiseless, because the signal-to-noise ratio (SNR) of the deformation measurements is much lower than the SNR of the force measurements for stiff payloads. Total least squares techniques [40], [41], unlike standard least squares techniques, are robust against noise on both the output variable and the regressor vector.

Second, the logarithmic transformation introduces an implicit weighting of the measurements. This can be shown as follows. By substituting $\delta_{k}$ and $F_{k}$ in equation (20) using equations (16)-(17), which represent realistic noise assumptions, the model can be rewritten as

$$
\ln \left(\hat{F}_{k}-\Delta F_{k}\right)=l+n \ln \left(\hat{\delta}_{k}-\Delta \delta_{k}\right),
$$

By series expansion of the logarithmic terms around $\Delta \delta_{k}=0$ and $\Delta F_{k}=0$ respectively, and retaining only the first order terms, equation (21) can be approximated as

$$
\ln \left(\hat{F}_{k}\right)-\frac{\Delta F_{k}}{\hat{F}_{k}}=l+n\left(\ln \left(\hat{\delta}_{k}\right)-\frac{\Delta \delta_{k}}{\hat{\delta}_{k}}\right) .
$$

Thus, both $\ln \left(F_{k}\right)$ and $\ln \left(\delta_{k}\right)$ are corrupted with zero-mean heteroskedastic ${ }^{4}$ noise with unequal variances $\left(\sigma^{F} / \hat{F}_{k}\right)^{2}$ and $\left(\sigma^{\delta} / \hat{\delta}_{k}\right)^{2}$ respectively.

Based on these considerations, the following errors-invariables model [40], [41] is proposed

$$
\ln \left(\hat{F}_{k}\right)=l+n\left(\ln \left(\hat{\delta}_{k}\right)+\Delta \ln \left(\delta_{k}\right)\right)+\Delta \ln \left(F_{k}\right),
$$

where the pseudo-measurements $\ln \left(\hat{\delta}_{k}\right)$ and $\ln \left(\hat{F}_{k}\right)$ are assumed to be corrupted by zero-mean noise $\Delta \ln \left(\delta_{k}\right)$ and

\footnotetext{
${ }^{4}$ Homoskedastic noise has a constant variance, as opposed to heteroskedastic noise, which has a non-constant variance.
}

$\Delta \ln \left(F_{k}\right)$ with variances $\left(\sigma^{\delta} / \hat{\delta}_{k}\right)^{2}$ and $\left(\sigma^{F} / \hat{F}_{k}\right)^{2}$ as in the approximated model (22). The best unbiased estimate of the parameters of model (23) is given by the element-wise weighted mixed least squares-total least squares [40] solution, which minimizes

$$
\frac{1}{L} \sum_{k=0}^{L-1}\left[\frac{\left(\ln \left(\delta_{k}\right)-\ln \left(\hat{\delta}_{k}\right)\right)^{2}}{\left(\sigma^{\delta} / \hat{\delta}_{k}\right)^{2}}+\frac{\left(\ln \left(F_{k}\right)-\ln \left(\hat{F}_{k}\right)\right)^{2}}{\left(\sigma^{F} / \hat{F}_{k}\right)^{2}}\right],
$$

subject to constraint (20) for $k=0 \ldots L-1$. However, since this technique is iterative and has no recursive variant, this paper adopts a row-wise scaled total least squares (RSTLS) [40] approach with weights $w_{k}=\hat{\delta}_{k}^{2}$ instead, which still yields unbiased estimates and which minimizes

$$
\begin{gathered}
\frac{1}{L} \sum_{k=0}^{L-1}\left[\frac{\left(p_{k}-1\right)^{2}}{\left(\sigma^{\delta} / \hat{\delta}_{k}\right)^{2}}+\frac{\left(\ln \left(\delta_{k}\right)-\ln \left(\hat{\delta}_{k}\right)\right)^{2}}{\left(\sigma^{\delta} / \hat{\delta}_{k}\right)^{2}}\right. \\
\left.+\frac{\left(\ln \left(F_{k}\right)-\ln \left(\hat{F}_{k}\right)\right)^{2}}{\left(\sigma^{\delta} / \hat{\delta}_{k}\right)^{2}}\right]
\end{gathered}
$$

subject to $\ln \left(F_{k}\right)=l p_{k}+n \ln \left(\delta_{k}\right)$ for $k=0 \ldots L-1$. It is not necessary to incorporate $1 /\left(\sigma^{\delta}\right)^{2}$ in the weights, since it is a common factor for all terms. While the weights are only correct $^{5}$ for the terms with $\ln \left(\hat{\delta}_{k}\right)$, the heteroskedasticity of the noise $\Delta \ln \left(F_{k}\right)$ is also partially compensated.

In the presence of modeling errors, when model (2) is only an approximation, it can be shown that RS-TLS with weights $w_{k}=\hat{\delta}_{k}^{2}$ generally yields considerably smaller force errors over the whole range of interest compared to standard TLS or LS. This property is important in light of the potential deviations from the power law model discussed in Sections II-A, II-B and II-C.

The batch (RS-BTLS) and recursive variant (RS-RTLS) of the RS-TLS technique can be easily implemented by premultiplying both the output variable $\ln \left(\hat{F}_{k}\right)$ and the regressor vector $\left[1 \ln \left(\hat{\delta}_{k}\right)\right]^{T}$ with $\sqrt{w_{k}}=\hat{\delta}_{k}$ and applying standard batch TLS [40], [41] and recursive TLS [42] to these scaled quantities.

\section{STVF EXPERIMENTS}

To assess the feasibility and achievable accuracy of estimating contact parameters for stiff payloads using robotic hardware, experiments are carried out for a number of plastic (Acetron GP Acetal, $E=2.75 \cdot 10^{9} \mathrm{~N} / \mathrm{m}^{2}, \nu=0.3$ ) and aluminum (Aluminum 6061, $E=70 \cdot 10^{9} \mathrm{~N} / \mathrm{m}^{2}, \nu=0.33$ ) payloads with different geometries shown in Fig. 1.

The experiments are carried out with the Special Purpose Dexterous Manipulator (SPDM) Task Verification Facility (STVF) manipulator [43], [44] at the Canadian Space Agency (CSA). The STVF manipulator is a very stiff, six degreeof-freedom hydraulic robot shown in Fig. 2, which is used as a hardware-in-the-loop simulator for validation of space operations [43], [44].

\footnotetext{
${ }^{5}$ To obtain the best unbiased estimate, the terms $\left(p_{k}-1\right)^{2}$ should have an infinite weight, while the terms $\left(\ln \left(F_{k}\right)-\ln \left(\hat{F}_{k}\right)\right)^{2}$ should have a weight $1 /\left(\sigma^{F} / \hat{F}_{k}\right)^{2}$.
} 


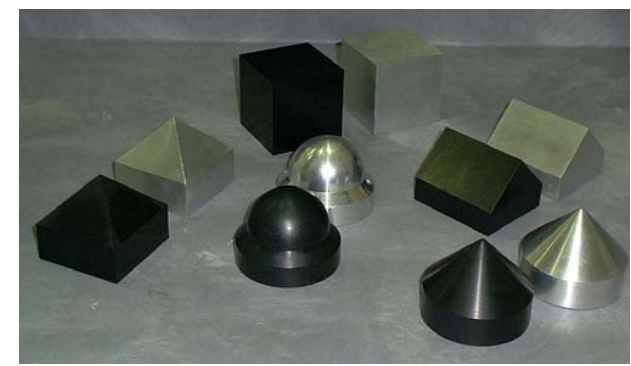

Fig. 1. Experimental payloads.

\section{A. Experimental setup and procedure}

The experimental setup consists of the STVF manipulator testbed (SMT) shown schematically in Fig. 3. The SMT is made up of the STVF manipulator, and its worksite equipped with a force plate on which an aluminum plate is mounted. A payload is mounted onto a micro-fixture which in turn is manipulated by the STVF manipulator using an end-effector gripper. The payload is oriented normal to the aluminum plate and brought into contact by a downward motion of the STVF end-effector. Subsequently, the contact force is increased in step-wise increments of $50 \mathrm{~N}$ up to $300 \mathrm{~N}$ and after each increment, the force is held constant for a few seconds. The deformation is calculated from the STVF endeffector displacement normal to the aluminum plate. The latter is in turn calculated from the forward rigid-body kinematics of the STVF manipulator, with an effective resolution of $\delta_{\Delta}=2 \cdot 10^{-6} \mathrm{~m}$. For typical robotic systems, $\delta_{\Delta}$ is often an order of magnitude larger. The displacement is measured relative to the end-effector position at the moment of first contact between the payload and the aluminum plate. The force component of the force plate normal to the aluminum plate is used as the force measurement, yielding a force resolution of $F_{\Delta}=1.5 \mathrm{~N}$. Both force and deformation measurements are collected at $1000 \mathrm{~Hz}$.

\section{B. System stiffness}

While the STVF manipulator and its worksite are very stiff, their combined stiffness is of the same order of magnitude

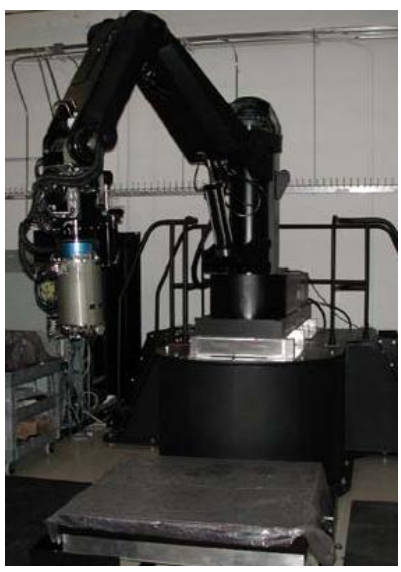

Fig. 2. STVF manipulator.

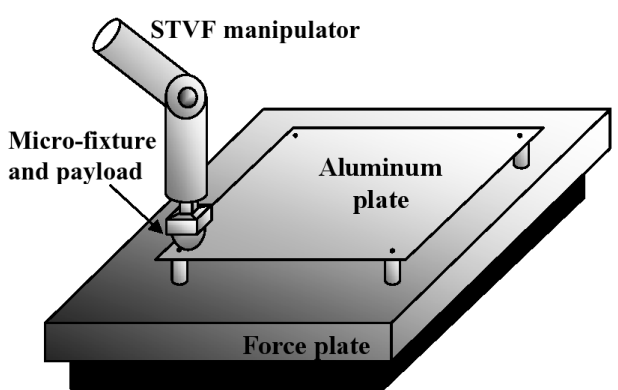

Fig. 3. STVF manipulator testbed (SMT).
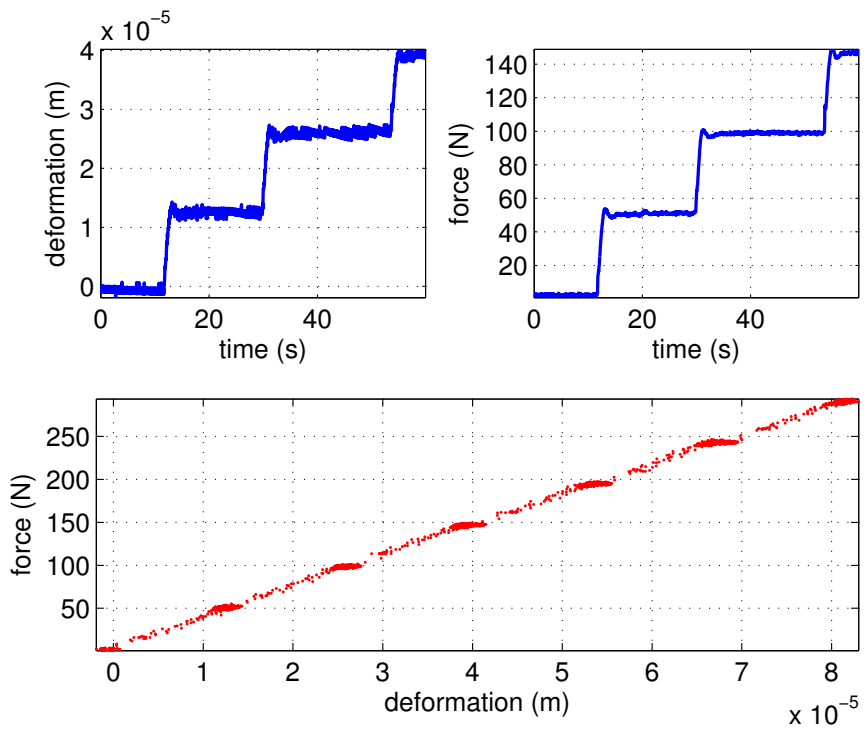

Fig. 4. The measured system deformation (top left) and contact force (top right) and the force-deformation relation (bottom) for the STVF manipulator and its worksite.

as the stiffness of some of the payloads. Therefore, the measured deformation calculated from the STVF rigid-body kinematics represents the combined deformation of the STVF manipulator, the worksite and the payload.

Therefore, first, an experiment is carried out to determine the stiffness of the STVF manipulator and the worksite, hereafter referred to as the system stiffness. To this end, similar experiments are carried out as discussed in Section IV-A, but without a payload attached to the micro-fixture. The measured deformation, the contact force, and the force-deformation relation are shown in Fig. 4, illustrating clearly that the deformation SNR $\delta_{\max } / \delta_{\Delta} \approx 40$ is notably lower than the force SNR $F_{\max } / F_{\Delta} \approx 200$ as mentioned in Section III. Because the force-deformation relation is very linear, the following model for the combined dynamics of the STVF manipulator and the worksite is adopted, which relates the force $F$ to the system deformation $\delta_{s}$ as

$$
F=K_{s} \delta_{s}+C_{s} \dot{\delta}_{s},
$$

The parameters $K_{s}$ and $C_{s}$ are calculated using a standard batch TLS algorithm. The obtained estimates are $\hat{K}_{s}=3.49$. $10^{6} \mathrm{~N} / \mathrm{m}$ and $\hat{C}_{s}=3.87 \cdot 10^{4} \mathrm{~N} \cdot \mathrm{s} / \mathrm{m}$. The damping term is included to reduce the influence of damping forces on the estimate of the system stiffness $\hat{K}_{s}$, but is not used in the 


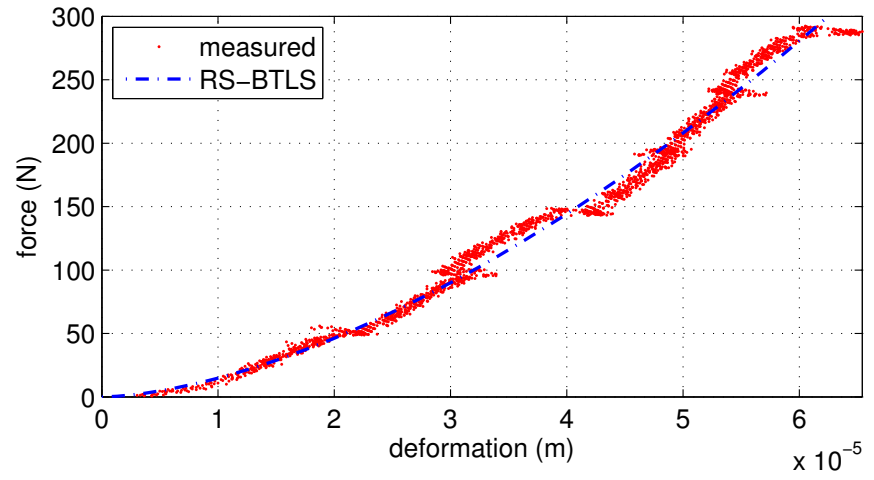

Fig. 5. The force-deformation relation for the plastic sphere (test 1).

remainder of this paper. The square root of the mean squared deformation error (MSDE) is $5.42 \cdot 10^{-7} \mathrm{~m}$, thereby confirming the linearity of the system stiffness. The MSDE characterizes the linearity of the system stiffness and it is a measure of the error introduced in calculating the payload deformation.

For the experiments conducted with the payloads, the payload deformation $\hat{\delta}_{p, k}$ at time-step $k$ is calculated from the measured deformation $\hat{\delta}_{k}$, the measured contact force $\hat{F}_{k}$ and the estimated system stiffness $\hat{K}_{s}$ as

$$
\hat{\delta}_{p, k}=\hat{\delta}_{k}-\hat{\delta}_{s, k}=\hat{\delta}_{k}-\frac{\hat{F}_{k}}{\hat{K}_{s}} .
$$

In the remainder of this paper, "deformation" and "measured deformation" are used to refer to the payload deformations calculated using equation (27), unless specified otherwise.

\section{Estimation results for the payload experiments}

Two experimental tests, named tests 1 and 2, are carried out with the STVF manipulator for the plastic sphere, cone, pyramid, prism and cube, and the aluminum sphere. The contact parameters are determined using both the batch (RS-BTLS) and recursive variant (RS-RTLS) of the algorithm proposed in Section III with the payload deformation and measured force as inputs. The results for the plastic and aluminum sphere are discussed in detail, while Table I summarizes the results of tests 1 and 2 for the plastic sphere, cone and pyramid, and the aluminum sphere.

For the batch estimation, the data is downsampled such that the force measurements are approximately uniformly distributed in the interval $[0,300] \mathrm{N}$ to avoid artificially overweighting the data collected during the times where the contact force is held constant. The measured relation between the payload deformation and the contact force and a best fit obtained with the RS-BTLS-estimates of the contact parameters are shown in Fig. 5 for the plastic sphere.

The measured force-deformation relation and a best fit using RS-BTLS for the aluminum sphere are shown in Fig. 6. The "discontinuous" appearance of the force-deformation relation is caused by the combination of kinematic errors and small end-effector motions in directions other than the normal to the aluminum plate, such as reorientation, at times when the contact force is held constant. The outliers for small

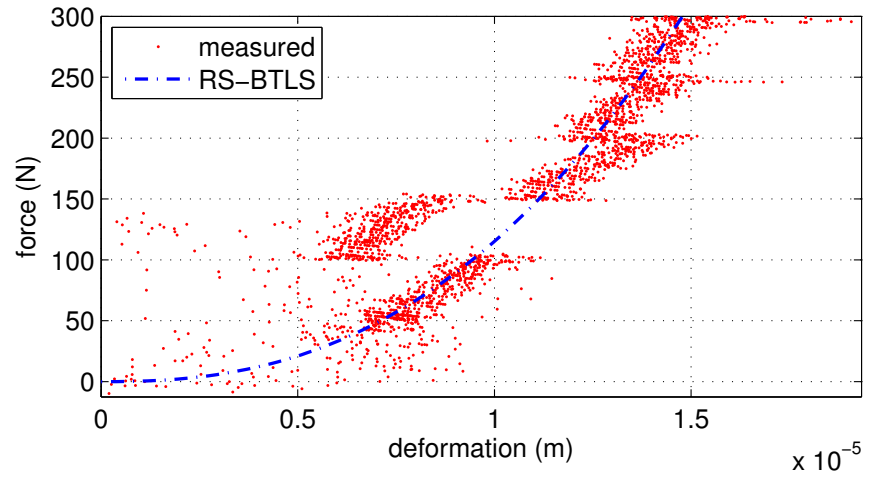

Fig. 6. The force-deformation relation for the aluminum sphere (test 1).

deformations are caused by instabilities during the making of contact. For the other payloads, contact was established in a stable manner.

Similar results to those for the plastic sphere are obtained for the other relatively compliant plastic payloads - the cone and the pyramid. Conversely, the measured force-deformation relations for the stiffer payloads - the plastic cube and the plastic prism - exhibit the discontinuities in the force-deformation relation even more pronounced than those in Fig. 6, and thus prohibit meaningful estimation.

For recursive estimation with RS-RTLS, the measurements are simply downsampled to $100 \mathrm{~Hz}$ and it is verified for the plastic sphere, cone and pyramid, and the aluminum sphere that the RS-RTLS algorithm is able to converge to the values determined by the RS-BTLS, despite the artificial weighting introduced by the periods of constant contact force. The results for the plastic sphere are shown in Fig. 7 (top left and right). To facilitate the interpretation, the secant stiffness $\underline{\hat{K}}$ for $F=$ $300 \mathrm{~N}$, which defines a linear relation at that load,

$$
\underline{\hat{K}}=\left(F^{(\hat{n}-1)} \hat{K}\right)^{(1 / \hat{n})}
$$
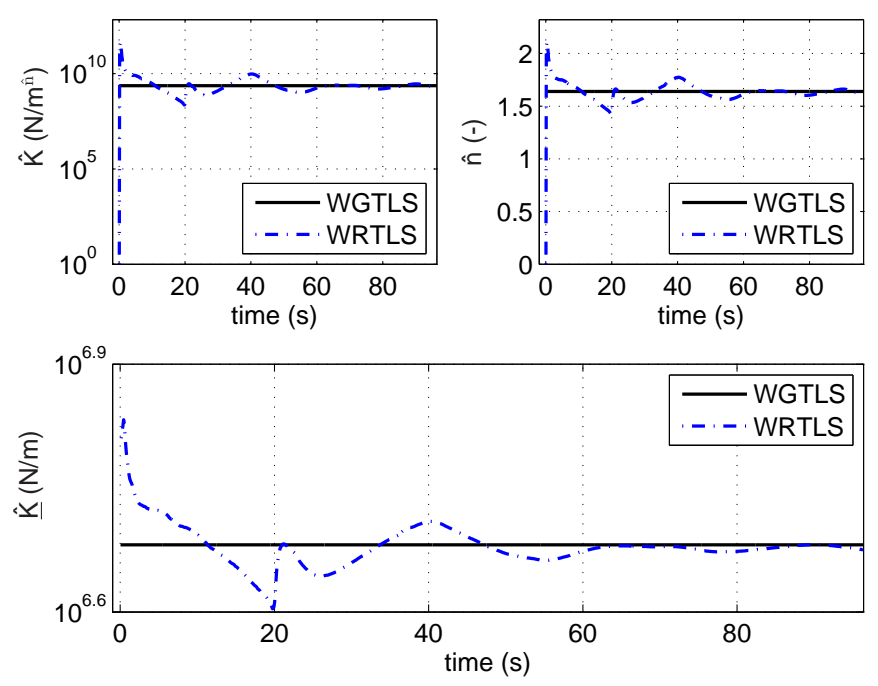

Fig. 7. The estimated stiffness $\hat{K}\left(\mathrm{~N} / \mathrm{m}^{\hat{n}}\right)$ (top left), exponent $\hat{n}$ (-) (top right), and calculated secant stiffness (bottom) $\underline{\hat{K}}(\mathrm{~N} / \mathrm{m})$ for the plastic sphere (test 1) using RS-BTLS (full line) and RS-RTLS (dash-dotted line). 
is also shown in Fig. 7 (bottom). The secant stiffness obtained with the RS-RTLS algorithm approaches the secant stiffness obtained with RS-BTLS within a few seconds. Since the deformation measurements are not only corrupted by zeromean noise, but also with some systematic errors such as kinematic errors, the estimated parameters using RS-RTLS converge closely to the RS-BTLS estimates only near the end. Furthermore, because a forgetting factor is used for RS-RTLS, and because of the different downsampling for RS-BTLS and RS-RTLS, the obtained parameter estimates are not exactly the same.

TABLE I

ESTIMATED PARAMETERS $\hat{K}\left(\mathrm{~N} / \mathrm{m}^{\hat{n}}\right)$ AND $\hat{n}(-)$, AND THE CALCULATED SECANT STIFFNESS $\underline{\hat{K}}(\mathrm{~N} / \mathrm{m})$ USING RS-BTLS AND RS-RTLS

\begin{tabular}{c|ccccc}
\cline { 3 - 6 } \multicolumn{2}{c}{} & \multicolumn{2}{c}{ Test 1 } & \multicolumn{2}{c}{ Test 2 } \\
\hline \hline Payload & & RS-BTLS & RS-RTLS & RS-BTLS & RS-RTLS \\
\hline \hline \multirow{2}{*}{$\begin{array}{c}\text { Plastic } \\
\text { sphere }\end{array}$} & $\hat{K}$ & $2.36 \cdot 10^{9}$ & $1.64 \cdot 10^{9}$ & $3.92 \cdot 10^{9}$ & $5.17 \cdot 10^{9}$ \\
\cline { 2 - 6 } & $\hat{n}$ & 1.64 & 1.60 & 1.70 & 1.72 \\
\cline { 2 - 6 } & $\hat{K}$ & $\mathbf{4 . 8 0 \cdot \mathbf { 1 0 } ^ { \mathbf { 6 } }}$ & $4.73 \cdot 10^{6}$ & $\mathbf{4 . 6 7} \cdot \mathbf{1 0}^{\mathbf{6}}$ & $4.75 \cdot 10^{6}$ \\
\hline \multirow{2}{*}{$\begin{array}{c}\text { Plastic } \\
\text { cone }\end{array}$} & $\hat{K}$ & $1.37 \cdot 10^{8}$ & $1.18 \cdot 10^{8}$ & $3.22 \cdot 10^{8}$ & $3.24 \cdot 10^{8}$ \\
\cline { 2 - 6 } & $\hat{n}$ & 1.48 & 1.47 & 1.57 & 1.57 \\
\cline { 2 - 6 } & $\hat{K}$ & $\mathbf{1 . 9 5} \cdot \mathbf{1 0}^{\mathbf{6}}$ & $1.93 \cdot 10^{6}$ & $\mathbf{2 . 0 7} \cdot \mathbf{1 0}^{\mathbf{6}}$ & $2.04 \cdot 10^{6}$ \\
\hline \multirow{2}{*}{$\begin{array}{c}\text { Plastic } \\
\text { pyramid }\end{array}$} & $\hat{K}$ & $1.03 \cdot 10^{7}$ & $9.32 \cdot 10^{6}$ & $4.97 \cdot 10^{7}$ & $1.27 \cdot 10^{8}$ \\
\cline { 2 - 6 } & $\hat{n}$ & 1.27 & 1.27 & 1.43 & 1.54 \\
\cline { 2 - 6 } & $\hat{K}$ & $\mathbf{1 . 0 9} \cdot \mathbf{1 0}^{\mathbf{6}}$ & $1.06 \cdot 10^{6}$ & $\mathbf{1 . 3 8} \cdot \mathbf{1 0}^{\mathbf{6}}$ & $1.37 \cdot 10^{6}$ \\
\hline \multirow{2}{*}{$\begin{array}{c}\text { Aluminum } \\
\text { sphere }\end{array}$} & $\hat{K}$ & $2.00 \cdot 10^{14}$ & $3.51 \cdot 10^{10}$ & $1.52 \cdot 10^{16}$ & $6.06 \cdot 10^{13}$ \\
\cline { 2 - 6 } & $\hat{n}$ & 2.45 & 1.68 & 2.81 & 2.33 \\
\cline { 2 - 6 } & $\hat{K}$ & $\mathbf{2 . 0 3} \cdot \mathbf{1 0}^{\mathbf{7}}$ & $1.86 \cdot 10^{7}$ & $\mathbf{2 . 2 5} \cdot \mathbf{1 0}^{\mathbf{7}}$ & $2.33 \cdot 10^{7}$ \\
\hline
\end{tabular}

The estimated contact parameters for all payloads obtained with the batch and the recursive algorithm at the final timestep, are summarized in Table I for tests 1 and 2. It is important to note that small perturbations in the force-deformation relation can give rise to considerable and simultaneous changes in the parameter values, such that the parameters $\hat{K}$ and $\hat{n}$ cannot easily be interpreted separately. Therefore, to facilitate interpretation, the secant stiffnesses are also given in Table I. Furthermore, because of the coupling between $\hat{K}$ and $\hat{n}$, care should be taken if using the parameters outside the range in which they were measured.

For the plastic sphere, cone and pyramid, $1 \leq \hat{n} \leq 2$. For both the plastic sphere and especially for the aluminum sphere (sphere radii $R=28.7 \cdot 10^{-3} \mathrm{~m}$ ), $\hat{n}$ and $\hat{K}$ differ notably from the Hertzian values of $n=3 / 2$, and $K=6.57 \cdot 10^{8} \mathrm{~N} / \mathrm{m}^{3 / 2}$ and $K=8.87 \cdot 10^{9} \mathrm{~N} / \mathrm{m}^{3 / 2}$ respectively. In light of the nonidealities discussed in Sections II-B and II-C, deviations from the theoretical values are not entirely unexpected.

For the cone and the pyramid, no analytical results are available for perfectly elastic contact. However, the Hertzian values for spherical contact based on the radius of curvature of the tip of these payloads (radii $R=3.3 \cdot 10^{-3} \mathrm{~m}$ and $R=1.15 \cdot 10^{-3} \mathrm{~m}$ respectively), provide an indication of the order of magnitude to be expected, namely $n=3 / 2$ and $K=2.23 \cdot 10^{8} \mathrm{~N} / \mathrm{m}^{3 / 2}$ and $K=1.32 \cdot 10^{8} \mathrm{~N} / \mathrm{m}^{3 / 2}$.

The secant stiffnesses obtained using RS-BTLS and RS-
RTLS typically match within a few percent within each test. For the plastic cone and pyramid, and the aluminum sphere, however, the secant stiffnesses increase considerably from the first to the second test, namely by $6 \%$ for the plastic cone, by $27 \%$ for the plastic pyramid and by $11 \%$ for the aluminum sphere. This is further discussed in Section V-C.

\section{COMPARISON OF THE STVF RESUlTS}

The RS-TLS algorithm presented in Section III yields a good power law fit of the measurements. However, the main question of this paper, whether or not the parameter estimates yield a force-deformation relation close to the true relation, is yet unanswered. To assess the validity of the STVF results, they are compared with a set of separate experiments in Section V-A and with finite element models in Section V-B.

\section{A. Instron experiments}

A set of contact experiments is carried out using a one-axis Instron material testing machine. The payloads are mounted onto a steel $\left(E=200 \cdot 10^{9} \mathrm{~N} / \mathrm{m}^{2}, \nu=0.29\right)$ platen and compressed by lowering a second steel platen. The force resolution of the Instron machine is $1 \mathrm{~N}$, while the position resolution is $1 \cdot 10^{-6} \mathrm{~m}$. For each payload, the best fit is calculated with RS-BTLS on the averaged data from a number of tests. Similarly to the STVF experiments, the system stiffness, in this case the stiffness of the Instron machine, is determined to be $\hat{K}_{s}=1.62 \cdot 10^{7} \mathrm{~N} / \mathrm{m}$. The square root of the corresponding MSDE between the fitted and measured relation is $1.31 \cdot 10^{-6} \mathrm{~m}$, indicating a weaker linearity for the Instron machine than for the STVF manipulator and its worksite.

Unlike in [19], [20], [31], where the Instron results are presented without taking into account the system stiffness, here we compensate for the system stiffness similarly to the STVF experiments. In addition, since the Instron experiments

TABLE II

ESTIMATED PARAMETERS $\hat{K}\left(\mathrm{~N} / \mathrm{m}^{\hat{n}}\right)$ AND $\hat{n}(-)$, AND THE CALCULATED SECANT STIFFNESS $\underline{\hat{K}}(\mathrm{~N} / \mathrm{m})$ USING RS-BTLS FOR THE FINITE ELEMENT MODELS (FEM) WITHOUT AND WITH CORRECTION FOR THE EQUIVALENT ROUGHNESS $\sigma_{\text {eq }}(\mu \mathrm{m})$ AND THE STVF (TEST 2) AND INSTRON EXPERIMENTS

\begin{tabular}{c|ccccc}
\hline Payload & & $\begin{array}{c}\text { STVF } \\
\text { experiments }\end{array}$ & $\begin{array}{c}\text { Instron } \\
\text { experiments }\end{array}$ & FEM & $\begin{array}{c}\text { FEM } \\
\left(\sigma_{\text {eq }}=7.5\right)\end{array}$ \\
\hline \hline \multirow{2}{*}{$\begin{array}{c}\text { Plastic } \\
\text { sphere }\end{array}$} & $\hat{K}$ & $3.92 \cdot 10^{9}$ & $3.85 \cdot 10^{10}$ & $6.16 \cdot 10^{8}$ & $1.82 \cdot 10^{10}$ \\
\cline { 2 - 6 } & $\hat{n}$ & 1.70 & 1.94 & 1.49 & 1.85 \\
\cline { 2 - 6 } & $\hat{K}$ & $\mathbf{4 . 6 7} \cdot \mathbf{1 0}^{\mathbf{6}}$ & $\mathbf{4 . 6 1} \cdot \mathbf{1 0}^{\mathbf{6}}$ & $\mathbf{5 . 0 5} \cdot \mathbf{1 0}^{\mathbf{6}}$ & $\mathbf{4 . 8 1} \cdot \mathbf{1 0}^{\mathbf{6}}$ \\
\hline \multirow{2}{*}{$\begin{array}{c}\text { Plastic } \\
\text { cone }\end{array}$} & $\hat{K}$ & $3.22 \cdot 10^{8}$ & $1.23 \cdot 10^{9}$ & $1.49 \cdot 10^{8}$ & $6.36 \cdot 10^{8}$ \\
\cline { 2 - 6 } & $\hat{n}$ & 1.57 & 1.70 & 1.46 & 1.63 \\
\cline { 2 - 6 } & $\hat{K}$ & $\mathbf{2 . 0 7} \cdot \mathbf{1 0}^{\mathbf{6}}$ & $\mathbf{2 . 3 5} \cdot \mathbf{1 0}^{\mathbf{6}}$ & $\mathbf{2 . 3 6} \cdot \mathbf{1 0}^{\mathbf{6}}$ & $\mathbf{2 . 3 1} \cdot \mathbf{1 0}^{\mathbf{6}}$ \\
\hline \multirow{2}{*}{$\begin{array}{c}\text { Plastic } \\
\text { pyramid }\end{array}$} & $\hat{K}$ & $4.97 \cdot 10^{7}$ & $8.41 \cdot 10^{7}$ & $4.17 \cdot 10^{7}$ & $9.64 \cdot 10^{7}$ \\
\cline { 2 - 6 } & $\hat{n}$ & 1.43 & 1.47 & 1.38 & 1.48 \\
\cline { 2 - 6 } & $\hat{K}$ & $\mathbf{1 . 3 8} \cdot \mathbf{1 0}^{\mathbf{6}}$ & $\mathbf{1 . 5 0} \cdot \mathbf{1 0}^{\mathbf{6}}$ & $\mathbf{1 . 5 6} \cdot \mathbf{1 0}^{\mathbf{6}}$ & $\mathbf{1 . 5 4} \cdot \mathbf{1 0}^{\mathbf{6}}$ \\
\hline Aluminum & $\hat{K}$ & $1.52 \cdot 10^{16}$ & $7.03 \cdot 10^{21}$ & $1.00 \cdot 10^{10}$ & $1.29 \cdot 10^{21}$ \\
\cline { 2 - 6 } sphere & $\hat{n}$ & 2.81 & 3.93 & 1.51 & 3.85 \\
\cline { 2 - 6 } & $\hat{K}$ & $\mathbf{2 . 2 5} \cdot \mathbf{1 0}^{\mathbf{7}}$ & $\mathbf{2 . 5 5} \cdot \mathbf{1 0}^{\mathbf{7}}$ & $\mathbf{2 . 8 8} \cdot \mathbf{1 0}^{\mathbf{7}}$ & $\mathbf{2 . 0 5} \cdot \mathbf{1 0}^{\mathbf{7}}$ \\
\hline & & & & & \\
\hline
\end{tabular}




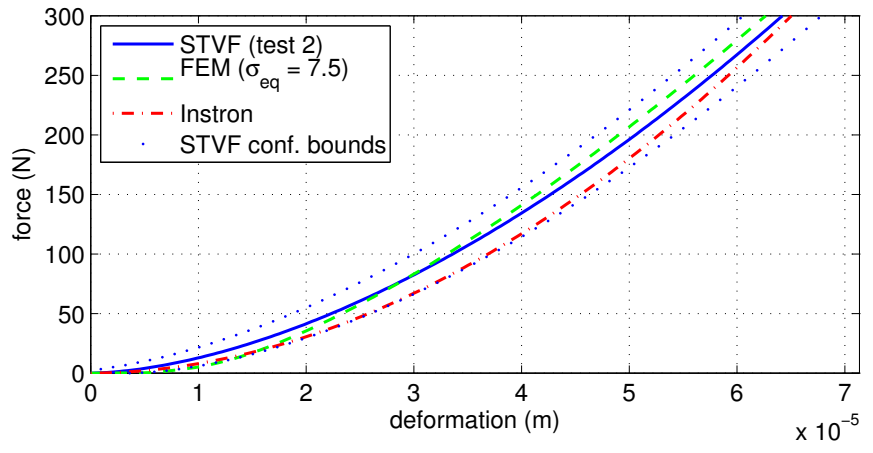

Fig. 8. The force - deformation relation of the plastic sphere for the STVF (test 2) and Instron experiments, and calculated using finite element modeling (FEM) with the correction for the roughness $\sigma_{\text {eq }}(\mu \mathrm{m})$.

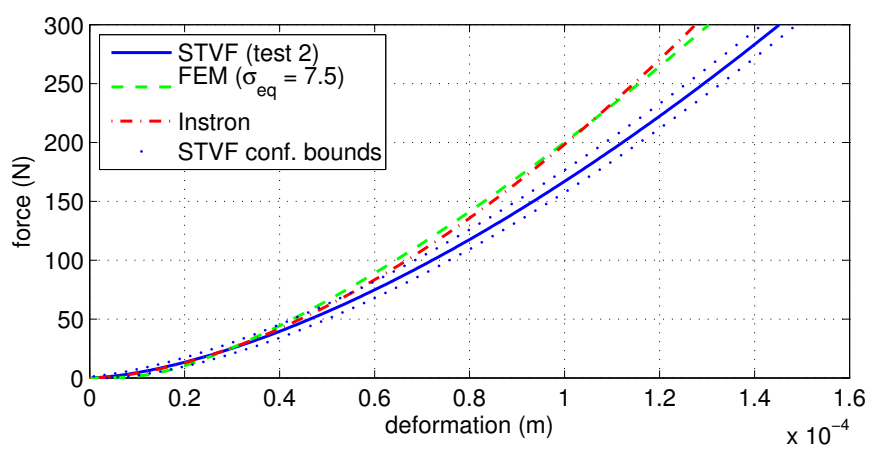

Fig. 9. The force - deformation relation of the plastic cone for the STVF (test 2) and Instron experiments, and calculated using finite element modeling (FEM) with the correction for the roughness $\sigma_{\text {eq }}(\mu \mathrm{m})$.

involve contact of the payloads with a steel platen while the STVF experiments are based on contact of payloads with an aluminum plate, a correction is implemented to the Instron results based on the ratio of the equivalent Young's moduli for aluminum/plastic - aluminum and aluminum/plastic - steel combinations.

The Instron best fit results with the above correction and the STVF best fit results for test 2 using RS-BTLS for the plastic sphere, cone and pyramid, and the aluminum sphere are shown in Figs. 8, 9, 10 and 11 respectively. Also shown are estimated confidence bounds for the STVF results, which are based on the resolution of the deformation measurements and the MSDE of the system stiffness. The confidence bounds for the Instron results are approximately of the same magnitude as the STVF confidence bounds, but are omitted for clarity. In addition, the estimated parameter values for the STVF and Instron experiments are summarized in Table II.

\section{B. Finite element modeling}

The experimental payloads in contact with an aluminum plate are also modeled using the ANSYS finite element software taking into account the local radii of curvature for the sharp payloads such as the cone and the pyramid. For the plastic and aluminum sphere, the finite element results are very close to the results given by Hertz's theory (maximum relative force error less than $0.2 \%$ ), while the finite element results for the plastic cone and plastic pyramid are reasonably close to the

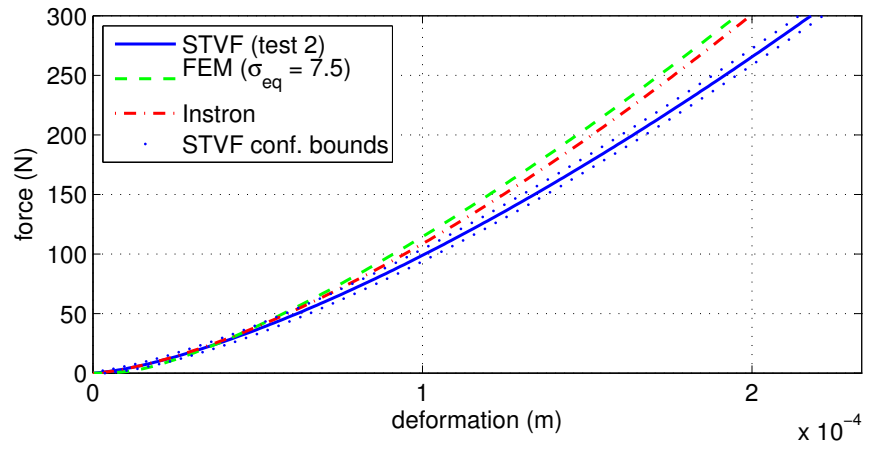

Fig. 10. The force - deformation relation of the plastic pyramid for the STVF (test 2) and Instron experiments, and calculated using finite element modeling (FEM) with the correction for the roughness $\sigma_{\text {eq }}(\mu \mathrm{m})$.

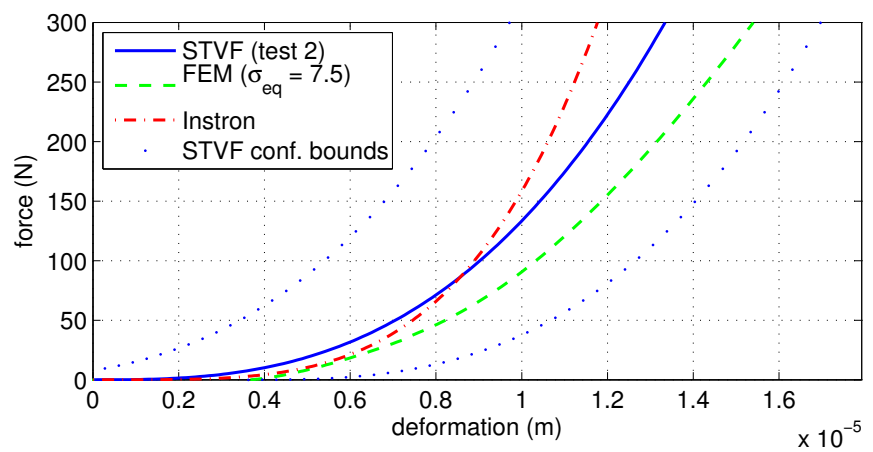

Fig. 11. The force - deformation relation of the aluminum sphere for the STVF (test 2) and Instron experiments, and calculated using finite element modeling (FEM) with the correction for the roughness $\sigma_{\text {eq }}(\mu \mathrm{m})$.

results predicted by Hertz's theory for spherical contact, where only the radius of curvature of the tip is taken into account (maximum relative force error $6 \%$ and $13 \%$ respectively).

To account for the surface roughness, the deformations of the finite element models are corrected using the multiplicative factor in equation (10). Since model (8)-(10) is only applicable to spheres, the correction factor is calculated using the force from the finite element models and taking into account only the radius of curvature of the tip for the cone and the pyramid ${ }^{6}$. The surface roughness for the payloads is determined as $\sigma=$ $\sqrt{\frac{\pi}{2}} R_{a}=7.5 \mu \mathrm{m}$, based on a crude estimate of the average roughness of $R_{a}=6 \mu \mathrm{m}$ resulting from the machining process to produce the payloads. The correction is relevant, although not very large for the plastic cone and pyramid, significant for the plastic sphere and very large for the aluminum sphere. The force-deformation relation for the aluminum sphere for the STVF experiments (tests 1 and 2) is compared with the finite element model with and without the correction for the roughness $\sigma_{\text {eq }}$ in Fig. 12.

The force-deformation relations for the STVF (test 2) and Instron experiments are compared with the finite element models with the correction for the roughness $\sigma_{\text {eq }}$ in Figs. 8, 9, 10

${ }^{6}$ The effect of the roughness is mainly important for small deformations, such that the calculation of the correction factor based on the spherical approximation of the cone and pyramid is reasonably accurate. For larger deformations, where the conical or pyramidical geometry becomes important, the correction factor tends to 1 , thus largely reducing the impact of the spherical approximation. 


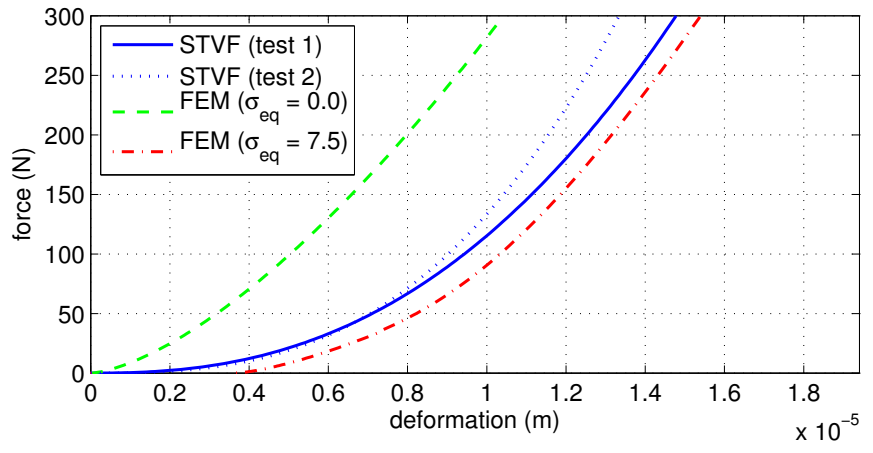

Fig. 12. The force - deformation relation of the aluminum sphere for the STVF experiments (tests 1 and 2) and calculated using finite element modeling (FEM) with and without the correction for the roughness $\sigma_{\text {eq }}(\mu \mathrm{m})$.

and 11. The estimated parameter values obtained using RSBTLS for the finite element models with and without the correction for the roughness $\sigma_{\mathrm{eq}}$, are summarized in Table II.

\section{Discussion}

For the sake of clarity, the experimental and finite element modeling results are discussed on a per payload basis.

Plastic sphere: Considering the confidence bounds shown in Fig. 8, there is a good agreement between the Instron and STVF experiments and the finite element model. Although not shown explicitly, the effect of the surface roughness is significant. The plastic sphere is not expected to exhibit elasticplastic behavior, as the maximum force applied in the STVF experiments is clearly below the maximum elastic force $F_{c}$ of equation (13), included in Table III.

TABLE III

THE MAXIMUM ELASTIC FORCE $F_{c}(\mathrm{~N})$ FOR THE PLASTIC SPHERE, CONE AND PYRAMID, AND THE ALUMINUM SPHERE.

\begin{tabular}{ccccc}
\cline { 2 - 4 } & $\begin{array}{c}\text { Plastic } \\
\text { sphere }\end{array}$ & $\begin{array}{c}\text { Plastic } \\
\text { cone }\end{array}$ & $\begin{array}{c}\text { Plastic } \\
\text { pyramid }\end{array}$ & $\begin{array}{c}\text { Aluminum } \\
\text { sphere }\end{array}$ \\
\hline$F_{c}$ & 727 & 9.6 & 1.2 & 261 \\
\hline
\end{tabular}

Plastic cone: The Instron experiment and the finite element model are in very good agreement (Fig. 9), while the agreement of these results with the STVF experiment is moderate and clearly outside the confidence bounds.

Here, we put forward the following explanation. From Table III, it can be seen that the maximum force applied to the cone in the STVF experiments exceeds $F_{c}$ by a factor of more than 30. Therefore, we expect time-varying behavior due to elastic-plastic effects discussed in Section II-C between STVF test 1 and test 2 and the Instron experiments, the latter being carried out last. The deformation calculated using the finite element model can be corrected to account for this behavior in a similar way as for the surface roughness using model (15), where the multiplicative factor is determined using the force calculated by the finite element model with the roughness correction, and taking into account only the radius of curvature of the tip of the cone.

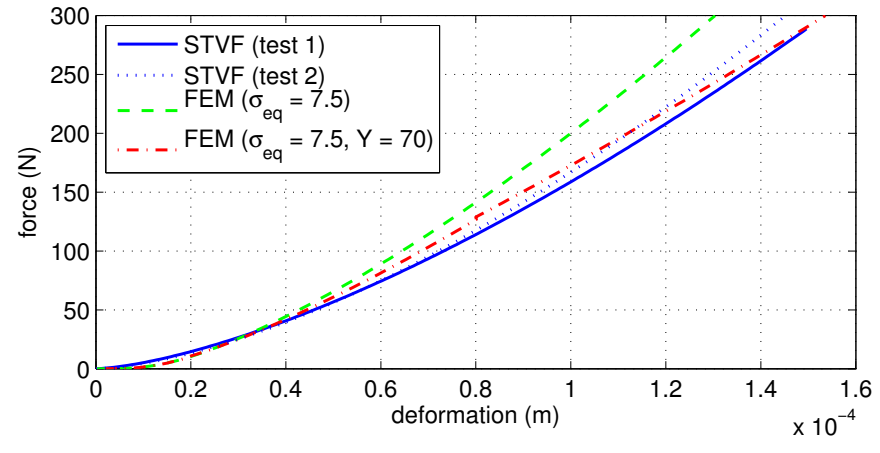

Fig. 13. The force - deformation relation of the plastic cone for the STVF experiments (test 1 and 2) and calculated using finite element modeling (FEM) with the correction for the elastic-plastic behavior $\left(Y\right.$ in $\left.10^{6} \mathrm{~N} / \mathrm{m}^{2}\right)$ and for the roughness $\sigma_{\text {eq }}(\mu \mathrm{m})$.

The force-deformation relations of the plastic cone for the STVF experiments (tests 1 and 2) and for the finite element model with the correction for the roughness, and in addition the correction for the elastic-plastic behavior (denoted with the yield stress $Y=70 \cdot 10^{6} \mathrm{~N} / \mathrm{m}^{2}$ of the plastic), are shown in Fig. 13. It can be seen that model (15) is able to explain the STVF experiments reasonably well.

Moveover, in agreement with [39], after a number of loading-unloading cycles, the behavior becomes fully elastic, which explains why the Instron experiment is in good agreement with the finite element model based on fully elastic assumptions (Fig. 9). In contrast, the fully elastic behavior is not yet reached for test 2 of the STVF experiments, as the result is in between the results from test 1 and the Instron experiment. This can be seen by comparing Figs. 9 and 13 and the secant stiffnesses in Tables I and II for the plastic cone.

Plastic pyramid: The discussion of the results is completely parallel to that for the plastic cone and is therefore omitted.

Aluminum sphere: The discrepancy between the STVF and Instron experiments is fairly large (Fig. 11), yet acceptable, given the confidence bounds. Although the bulk material of the aluminum sphere is not expected to exhibit considerable elastic-plastic behavior, the asperities which make up the rough sphere surface, are prone to deform plastically, even for small loads. While test 1 of the STVF experiments matches well with the finite element model corrected for the roughness (Fig. 12), the force-deformation relation seems to become increasingly stiffer for test 2 and for the Instron experiment as can be seen in Figs. 11 and 12. However, considering the confidence bounds for the experiments, it is difficult to reliably distinguish between the effect of this time-varying behavior and experimental errors.

In summary, for the plastic payloads, the force-deformation relations identified from the STVF and Instron experiments agree well with the finite element models, provided that effects such as plastic deformation and roughness are taken into account. This is also supported by the agreement of the parameter estimates $\hat{n}$, the secant stiffnesses $\underline{\hat{K}}$ and the order of magnitude of the parameter estimates $\hat{K}$, which is typically within or close to $10 \%$. The difference between the STVF and 
Instron experiments can be largely attributed to time-varying behavior and experimental error sources. Conversely, for the aluminum sphere, to the best of the author's knowledge, no models exist which are able to describe the effect of plastic deformation of the rough surface itself and the impact of experimental errors may be rather large. However, given the confidence bounds, the agreement of the STVF and Instron experiments with the finite element model is still reasonable. This is also supported by the agreement of the parameter estimates $\hat{n}$, the secant stiffnesses $\underline{K}$ and the order of magnitude of the parameter estimates $\hat{K}$, which is close to $25 \%$.

\section{ERROR SOURCES}

The differences between the Instron and STVF experiments illustrate that there is an unavoidable limitation in the accuracy of the identified force-deformation relations, which depends on the hardware and the procedure used for the identification. Here, we point out a number of contributing error sources which are inevitably present in robotic experiments and classify them into four categories: experiment design (D), robotic system $(\mathrm{S})$, data processing $(\mathrm{P})$ and uncontrolled variations in the environment $(\mathrm{E})$.

The major experiment design related error source is: (D1) the choice of the maximum contact force: If the force applied to a payload exceeds the maximum elastic force $F_{c}$, permanent changes in the force-deformation relation can occur. As shown in Table III, for sharp payloads, this force may only be a few Newtons. Furthermore, for payloads with rough surfaces, even if the force does not exceed $F_{c}$, changes in the force-deformation may easily occur, due to evolution of the roughness profile by plastic deformation of asperities. Therefore, the choice of the maximum contact force affects the magnitude of variations in the contact dynamics response, especially for sharp and/or rough payloads.

Experimental error sources related to the robotic system are: (S1) the limited sensor resolution of the robotic system.

(S2) the coupled kinematics and sensors for multi-axis systems: Since position measurements for multi-axis systems are typically calculated based on rigid-body kinematics using sensor measurements of the individual axes, the magnitude of the position measurement errors depends on the position of the individual axes and is often larger than visible variations due to quantisation errors. For multi-axis robotic systems, quantisation errors, inaccurately known kinematics and mechanical deficiencies, may all add up and cause significant errors.

(S3) geometric errors of the robotic system with respect to the worksite and the payload: Contact experiments require contact between the robotic system and its worksite. However, the location of the worksite with respect to the robotic system is typically not known very accurately. Furthermore, geometric errors between the payload and the end-effector due to gripping or fixture inaccuracies are also typically present. Therefore, the orientation of the end-effector and the payload with respect to the worksite, and the location of the contact point on the payload and the worksite, may not be accurately known and may vary during the experiment.

(S4) the use of the system stiffness to calculate the payload deformations: The measurement of the system stiffness, which is needed to calculate the payload deformations, is evidently subject to the same error sources (S1), (S2) and (S3). In addition, since contact is required to measure the system dynamics, the measured dynamics consist not only of the structural dynamics of the robotic system and its worksite, but also of the dynamics of the contact established to determine the system dynamics. This source of contact dynamics is not present in the experiments with the payloads, because the payloads are used to establish the contact with the worksite. Furthermore, while the system dynamics are measured in a certain position/configuration, they may need to be extrapolated to other positions, because the payload dimensions affect the endeffector position at which contact is made with the worksite. Moreover, because it is difficult to estimate damping and mass parameters for stiff system dynamics, typically, it is only possible to use the quasi-static system dynamics to calculate the payload deformations. Furthermore, this paper limits itself to a uniaxial approximation of the system dynamics, which are in general six-dimensional.

( $\left.S^{*}\right)$ Other error sources: In addition to the above sources, numerous other sources related to the robotic system can be mentioned. However, based on the experimental investigations, the authors believe (S4), (S2), (S3) and (S1) to be the most important systematic error sources related to the robot system. With regard to (S4), it is found that the system dynamics are best represented by a linear model, because structural dynamics are typically reasonably linear and the linear model allows to rule out some of the irreproducibility due to the presence of the contact dynamics in the measurement of the system dynamics.

The major data processing related error source is: $(P 1)$ the choice of the reference position with respect to which the deformations are calculated: For very stiff payloads, the force-deformation relation is quite sensitive to the choice of the reference position. This is especially the case for contact between rough surfaces, where the force onset is very slow, as for the aluminum sphere in Fig. 11.

Finally, we cite a number of error sources related to changes in the environment (E). Examples include:

(E1) geometric variations due to temperature changes: These variations are typically around $10^{-6} \mathrm{~m}$ per degree.

(E2) physical or chemical changes in the payloads due to reactions such as oxidation. The effect of these sources, however, is usually considerably smaller than that of the above-mentioned error sources.

\section{LIMITS OF IDENTIFIABILITY}

Based on the experimental error sources pointed out in Section VI, it is possible to characterize the maximum identifiable stiffness parameter $K_{\max }$ in the power law model (2) for a given $n$, for a specific robotic system with position resolution $\delta_{\Delta}$, maximum exertable force $F_{\max }$ and system dynamics, characterized by the linear system stiffness $K_{s}$. As discussed in Section III, it is assumed that the SNR of the force measurements is considerably higher than the SNR of the deformation measurements, such that the force resolution $F_{\Delta}$ is not a limiting factor. 
Since the payload deformations are calculated by substracting the estimated system deformation, the error in the latter is characterized first. For a measured force $\hat{F}_{k}$, the estimated system deformation is $\hat{\delta}_{s, k}=\hat{F}_{k} / K_{s}$ and the accuracy of this estimate is limited by the error sources discussed in Section VI. The error in $\hat{F}_{k} / K_{s}$ introduced by (S1), (S2), and (P1) is typically bounded by a multiple $p$ of the position resolution $\delta_{\Delta}$. Conversely, the effects of damping and mass (S4), the misfit of the system stiffness due to nonlinearity (S4), and geometric errors (S3), are typically smaller than a fraction $1 / s$ of the maximum system deformation $F_{\max } / K_{s}$, such that the errors between the true $\delta_{s, k}$ and the estimated system deformation $\hat{F}_{k} / K_{s}$ are bounded by

$$
\left|\delta_{s, k}-\frac{\hat{F}_{k}}{K_{s}}\right| \lesssim \max \left\{p \delta_{\Delta}, \frac{F_{\max }}{s K_{s}}\right\},
$$

where $\lesssim$ is used to denote that the left hand side is usually smaller than the right hand side.

Similarly, the errors on the measured combined deformation $\hat{\delta}_{k}$ of the robotic system and the payload are caused by (S1), (S2) and (P1), and can also be bounded ${ }^{7}$ by a multiple $p$ of the position resolution $\delta_{\Delta}$, such that the error between the estimated payload deformation $\hat{\delta}_{k}-\hat{F}_{k} / K_{s}$ and the true payload deformation $\delta_{p, k}$, is bounded by the sum of a multiple of the position resolution and the errors in the estimated system deformation $\hat{F}_{k} / K_{s}$

$$
\left|\hat{\delta}_{k}-\frac{\hat{F}_{k}}{K_{s}}-\delta_{p, k}\right| \lesssim p \delta_{\Delta}+\max \left\{p \delta_{\Delta}, \frac{F_{\max }}{s K_{s}}\right\} .
$$

To succesfully identify the payload stiffness parameter $K$, the errors in the calculated payload deformation need to be lower than a fraction $1 / t$ of the maximum payload deformation $\left(F_{\max } / K\right)^{(1 / n)}$, such that

$$
p \delta_{\Delta}+\max \left\{p \delta_{\Delta}, \frac{F_{\max }}{s K_{s}}\right\} \lesssim \frac{1}{t}\left(\frac{F_{\max }}{K}\right)^{(1 / n)} .
$$

Rewriting inequality (31) as an upper bound for $K$ yields

$$
K \lesssim \frac{F_{\max }}{\left(\max \left\{2 p t \delta_{\Delta}, p t \delta_{\Delta}+\frac{t F_{\max }}{s K_{s}}\right\}\right)^{n}} .
$$

Summarizing, the maximum identifiable stiffness parameter $K_{\max }$ is approximately proportional to the right hand side of equation (32).

To facilitate the interpretation, inequality (32) can also be rearranged in terms of the secant stiffness $\underline{K}_{\max }=$ $\left(F_{\max }^{(n-1)} K_{\max }\right)^{(1 / n)}$ at $F_{\max }$, yielding a maximum identifiable linear or secant stiffness $\underline{K}_{\max }$ independent of $n$, namely

$$
\underline{K}_{\max } \sim \frac{s F_{\max } K_{s}}{\max \left\{2 p s t \delta_{\Delta} K_{s}, p s t \delta_{\Delta} K_{s}+t F_{\max }\right\}} .
$$

\footnotetext{
${ }^{7}$ In addition, there is the effect of geometric errors (S3) which can be bounded by a fraction of the combined maximum deformation of the robotic system and the payload. However, because (S3) is not an intrinsic limitation of the robotic system, and to simplify the discussion, (S3) is not included.
}

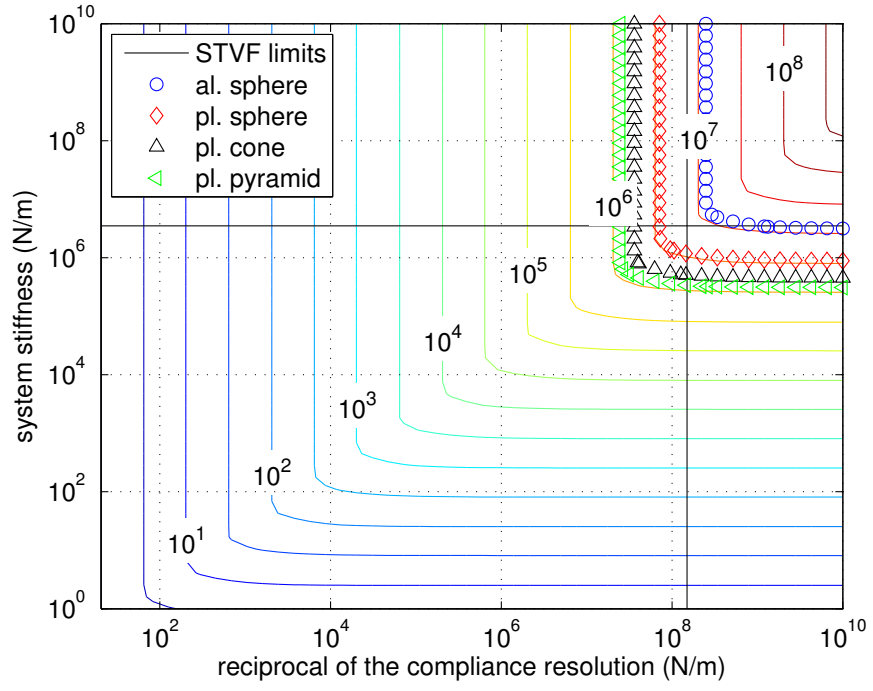

Fig. 14. Maximum identifiable payload secant stiffness as a function of the system stiffness and the reciprocal of the compliance resolution.

Introducing $K_{r}=F_{\max } / \delta_{\Delta}$, which is termed the reciprocal of the compliance resolution of the robotic system ${ }^{8}$, equation (33) can be rewritten as

$$
\underline{K}_{\max } \sim \frac{s K_{r} K_{s}}{\max \left\{2 p s t K_{s}, p s t K_{s}+t K_{r}\right\}} .
$$

The value of $\underline{K}_{\max }$ as a function of the system stiffness $K_{s}$ and the reciprocal of the compliance resolution $K_{r}$ is shown in Fig. 14 for typical values ${ }^{9}$ of $p=2, s=20$ and $t=5$, along with the STVF values of $K_{s}$ and $K_{r}$, and the secant stiffnesses for $F_{\max }=300 \mathrm{~N}$ for the plastic sphere, cone and pyramid, and the aluminum sphere. It can be seen that the aluminum sphere is actually outside the range of the identifiable stiffnesses for the STVF manipulator, while the plastic sphere is fairly close to the boundaries. The cube and the prism, for which analytical results [32] predict a secant stiffness of $\underline{K} \approx 2 \cdot 10^{8} \mathrm{~N} / \mathrm{m}$, are clearly beyond the maximum identifiable stiffness bound $\underline{K}_{\max } \sim 10^{6.9} \mathrm{~N} / \mathrm{m}$ for the STVF manipulator. For more common robot systems, where typically $K_{r} \sim 10^{6} \mathrm{~N} / \mathrm{m}$ and $K_{s} \sim 10^{5} \mathrm{~N} / \mathrm{m}, \underline{K}_{\max }$ is considerably lower and closer to $10^{5} \mathrm{~N} / \mathrm{m}$.

Fig. 14 also illustrates that, in order to identify stiff payloads, both the reciprocal of the compliance resolution and the system stiffness must be sufficiently high. If the system stiffness is too low, increasing the reciprocal of the compliance resolution may not suffice. Instead, a stiffer robotic system may be required to limit the absolute errors introduced by the linear approximation of the system dynamics. Alternatively, a more complicated nonlinear model of the system dynamics might also be used.

It is worth noting that the limit given by equation (34) is not a strict boundary, but rather a guideline for the feasibility of succesful identification. This guideline, however, does not pre-

\footnotetext{
${ }^{8}$ The compliance resolution $\delta_{\Delta} / F_{\max }$ is the smallest distinguishable difference between two compliances.

${ }^{9} t=5$ is chosen such that the maximum error on the calculated payload deformation $\hat{\delta}_{p, k}$ is less than $20 \%$ of the maximum payload deformation.
} 
vent succesful identification of higher stiffnesses. Indeed, the agreement of the finite element model with test 1 of the STVF experiments in Fig. 12 suggests that the force-deformation for the aluminum sphere is nevertheless succesfully identified.

Two important limit cases can be distinguished for equation (34). On the one hand, if $K_{r} \gg K_{s}$, or in other words, if the position resolution and the maximum exertable force of the robotic system are very high, the maximum identifiable linear payload stiffness is bounded by a multiple of the system stiffness $K_{s}$, namely

$$
\underline{K}_{\max } \sim \frac{s K_{s}}{t} .
$$

If the system deformation is not used to calculate the payload deformations, then $s=1$ in equation (35). Namely, assuming perfect position resolution, the maximum error on the payload deformation is equal to the maximum system deformation. In contrast, typically $s \sim 10$ and $s \lesssim 100$ if the system deformation is compensated. In other words, compensating for the system deformation increases the maximum identifiable payload stiffness by only an order of magnitude.

On the other hand, for a very high system stiffness $K_{s} \gg$ $K_{r}$, the maximum identifiable payload stiffness is logically bounded by a fraction of the reciprocal of the compliance resolution $K_{r}$

$$
\underline{K}_{\max } \sim \frac{K_{r}}{2 p t}
$$

where typically $p t \sim 10$. While equation (34) provides an indication of the maximum linear stiffness $\underline{K}_{\max }$ that can be identified with a relative accuracy related to $1 / t$, limit cases (35) and (36) provide an indication of the order of magnitude required for $K_{s}$ and $K_{r}$, and can hence be interpreted as, albeit crude, design guidelines.

\section{CONCLUSIONS AND FUTURE WORK}

This paper discusses the validity of the power law model and the effect of non-idealities on the contact dynamics model for stiff payloads. A row-wise scaled total least squares algorithm is applied to identify the contact parameters as this method can successfully deal with noise on the deformation measurements and modeling errors. Experimental results using a stiff six degree-of-freedom hydraulic robot are presented and compared to a separate set of experiments and finite element models.

The experimental results confirm the feasibility of identification of contact dynamics for a range of stiff payloads using practical robotic hardware. Moreover, the agreement of the experimental results with the modeling results shows that it is possible to obtain good estimates of the contact dynamics relation without necessarily having to resort to experiments, provided that non-ideal effects such as surface roughness and plastic deformation are taken into account. Unfortunately, the range of payloads for which results can be obtained using simple models, is mainly limited to spherical contact. For other payloads, linear statics analysis using finite element software is applicable if the surface roughness is not an important factor and if the applied load does not cause elastic-plastic behavior. If elastic-plastic behavior occurs, it may be necessary to resort to finite element modeling as in [37]-[39] where plastic behavior is taken into account.

In other cases, experiments are still necessary. Fortunately, the guidelines established in this paper provide a basis to evaluate the feasibility of identifying contact dynamics parameters for other payloads using another robotic system. In addition, they also allow to make experiment design decisions, if applicable, such as the geometry and material of the payloads, which allow succesful identification. Further work is required to investigate the limits of identifiability with other robotic systems. To this end, the guidelines can be used as a first prediction to make a suitable choice of payloads.

\section{ACKNOWLEDGMENTS}

The authors gratefully acknowledge the financial support by Prof. R. Snoeys Foundation, K.U.Leuven's Concerted Research Action GOA/05/10, K.U.Leuven's CoE EF/05/006 Optimization in Engineering Center (OPTEC), the Belgian Programme on Interuniversity Attraction Poles initiated by the Belgian Federal Science Policy Office, the Canadian Space Agency and Natural Sciences and Engineering Research Council of Canada (NSERC). The authors gratefully acknowledge Christian Lange, Yves Gonthier and Stefan Rondeau of the Canadian Space Agency and Julie Agar for conducting experiments with the STVF robotic facility and the Instron material testing machine.

\section{REFERENCES}

[1] M. K. Vukobratovic and P. Velkjo, "Dynamics of contact tasks in robotics. part I: general model of robot interacting with environment,' Mechanism and Machine Theory, vol. 34, no. 1, pp. 923-942, 1999.

[2] P. Velkjo and M. K. Vukobratovic, "Dynamics of contact tasks in robotics. part II: case study in dynamics of contact motion," Mechanism and Machine Theory, vol. 34, no. 1, pp. 943-972, 1999.

[3] J. Van Vliet, I. Sharf, and O. Ma, "Experimental validation of contact dynamics simulation of constrained robotic tasks," The International Journal of Robotics Research, vol. 19, pp. 1203-1217, 2000.

[4] O. Ma and R. Carr, "Dynamics modelling and simulation of SPDM contact operations, part I: Simulation model and berthing 6B avionics boxes," in Proceedings of the 1st IAF Space Robotics Workshop, SaintHubert, Québec, 1998.

[5] — "Dynamics modelling and simulation of SPDM contact operations, part II: SPDM handling the IEA battery ORUs," in Proceedings of the 1st IAF Space Robotics Workshop, Saint-Hubert, Québec, 1998.

[6] H. Hertz, "Über die berührung fester elastischer körper,' Journal für die reine und angewandte Mathematik, vol. 92, pp. 156-171, 1881.

[7] N. Hogan, "Impedance control: An approach to manipulation. Parts IIII," Transactions of the ASME, Journal of Dynamic Systems, Measurement, and Control, vol. 107, pp. 1-24, 1985.

[8] T. Yabuta, T. Yamada, T. Tsujimura, and H. Sakata, "Force control of servomechanism using adaptive control," IEEE Transactions on Robotics and Automation, vol. 4, no. 2, pp. 223-228, 1988.

[9] S.-T. Lin and H. K. Yae, "Identification of unknown payload and environmental parameters for robot compliant motion," in Proceedings of the American Control Conference, Chicago, 1992, pp. 2952-2956.

[10] S. K. Singh and D. O. Popa, "An analysis of some fundamental problems in adaptive control of force and impedance behavior: Theory and experiments," IEEE Transactions on Robotics and Automation, vol. 11, no. 1, pp. 912-921, 1995.

[11] L. Love and W. Book, "Environment estimation for enhanced impedance control," in Proceedings of the IEEE International Conference on Robotics and Automation, Nagoya, Japan, 1995.

[12] H. Seraji and R. Colbaugh, "Force tracking in impedance control," The International Journal of Robotics Research, vol. 16, no. 1, pp. 97-117, 1997. 
[13] S. Blouin, R. Hurteau, and R. Gourdeau, "Identification paramétrique d'un environnement par une tâche de contact d'un manipulateur robotique," in Proceedings of the International Conference on Industrial Automation, 1999, pp. 921-924.

[14] J. Park, R. Cortesão, and O. Khatib, "Robust and adaptive teleoperation for compliant motion tasks," in Proceedings of the International Conference on Advanced Robotics, Coimbra, Portugal, 2003, pp. 513-519.

[15] G. De Gersem, "Kinaesthetic feedback and enhanced sensitivity in robotic endoscopic telesurgery," Ph.D. dissertation, Katholieke Universiteit Leuven, Department of Mechanical Engineering, Belgium, 2005.

[16] R. Kikuuwe and T. Yoshikawa, "Robot perception of environment impedance," in Proceedings of the IEEE International Conference on Robotics and Automation, Washington DC, VA, 2002, pp. 1661-1666.

[17] — - "Recognizing surface properties using impedance perception," in Proceedings of the IEEE International Conference on Robotics and Automation, Taipeh, Taiwan, 2003, pp. 1539-1544.

[18] D. Erickson, "Contact stiffness and damping estimation for constrained robotic systems," Master's thesis, University of Victoria, Victoria, Canada, 2000

[19] J. Agar, "Contact parameter estimation using a space manipulator verification facility," Master's thesis, McGill University, Department of Mechanical Engineering, Montreal, Canada, 2004.

[20] J. Agar, I. Sharf, C. Lange, and Y. Gonthier, "Identification of contact dynamics model parameters from constrained robotic operations," in Proceedings of the ASME International Design Engineering Technical Conferences and Computers and Information in Engineering Conference. Long Beach, CA: ASME, 2005.

[21] M. Weber, "Contact parameter identification for constrained robotic tasks with known geometry," Master's thesis, University of Applied Sciences - Fachhochschule Ravensburg-Weingarten, 2001.

[22] M. Weber, O. Ma, and I. Sharf, "Identification of contact dynamics model parameters from constrained robotic operations," in Proceedings of the ASME International Design Engineering Technical Conferences and Computers and Information in Engineering Conference. Montreal, Canada: ASME, 2002.

[23] D. Erickson, M. Weber, and I. Sharf, "Contact stiffness and damping estimation for robotic systems," The International Journal of Robotics Research, vol. 22, no. 1, pp. 41-57, 2003.

[24] K. Patel, "Frequency and time domain contact parameter estimation for space robotic operations," Master's thesis, McGill University, Montreal, Canada, 2003

[25] C. Lange and Y. Gonthier, "Experimental contact parameter estimation using a 6-DOF robot," in Proceedings of the CISM-IFToMM Symposium on Robotics Design, Dynamics and Control, Montreal, Canada, 2004.

[26] N. Diolaiti, C. Melchiorri, and S. Stramigioli, "Contact impedance estimation for robotic systems," in Proceedings of the IEEE/RSJ International Conference on Intelligent Robots and Systems, Sendai, Japan, 2004, pp. 925-935.

[27] — - "Contact impedance estimation for robotic systems," IEEE Transactions on Robotics, vol. 21, no. 5, pp. 925-935, 2005.

[28] K. H. Hunt and F. R. E. Crossley, "Coefficient of restitution interpreted as damping in vibroimpact," Journal of Applied Mechanics, vol. 42, pp. 440-445, 1975.

[29] G. Gilardi and I. Sharf, "Literature survey of contact dynamics," Mechanism and Machine Theory, vol. 37, no. 1, pp. 1213-1239, 2002.

[30] Y. Gonthier, J. McPhee, C. Lange, and J.-C. Piedbœuf, "A regularized contact model with asymmetric damping and dwell-time dependent friction," Multibody Systems, vol. 11, pp. 209-233, 2004.

[31] X. Mu and I. Sharf, "Nonlinear contact parameter estimation for robotic systems with payload," in Proceedings of the ASME International Conference on Multibody Systems, Nonlinear Dynamics and Control, Las Vegas, NV, 2007.

[32] K. L. Johnson, Contact Mechanics. Cambridge: Cambridge University Press, 1985.

[33] H. M. Lankarani and P. E. Nikravesh, "Contact force model with hysteresis damping for impact analysis of multibody systems," Journal of mechanisms, transmissions, and automation in design, vol. 112, no. 3, pp. 369-376, 1990.

[34] G. Fu and A. Chandra, "Normal identation of elastic half-space with a rigid frictionless axisymmetric punch," Journal of Applied Mechanics, vol. 69, pp. 142-147, 2002.

[35] G. Fu, "An extension of Hertz's theory in contact mechanics," Journal of Applied Mechanics, vol. 74, pp. 373-374, 2007.

[36] M. Bahrami, M. M. Yovanovich, and J. R. Culham, "A compact model for spherical rough contacts," Journal of Tribology, vol. 127, pp. 884 889, 2005.
[37] L. Kogut and I. Etsion, "Elastic-plastic contact analysis of a sphere and a rigid flat," Journal of Applied Mechanics, vol. 69, pp. 657-662, 2002.

[38] I. Etsion, Y. Kligerman, and Y. Kadin, "Unloading of an elastic-plastic loaded spherical contact," International Journal of Solids and Structures, vol. 42, pp. 3716-3729, 2005.

[39] Y. Kadin, Y. Kligerman, and I. Etsion, "Multiple loading-unloading of an elastic-plastic spherical contact," International Journal of Solids and Structures, vol. 43, pp. 7119-7127, 2006.

[40] I. Markovsky and S. Van Huffel, "Overview of total least-squares methods," Signal Processing, vol. 87, no. 10, pp. 2283-2302, 2007.

[41] G. H. Golub and C. F. Van Loan, "An analysis of the total least squares problem,” SIAM Journal on Numerical Analysis, vol. 17, pp. 883-893, 1980.

[42] M. W. Soijer, "Sequential computation of total least squares parameter estimates," Journal of Guidance, Control and Dynamics, vol. 27, no. 3, pp. 501-503, 2004.

[43] J.-C. Piedbœuf, J. de Carufel, F. Aghili, and E. Dupuis, "Task verification facility for the Canadian special purpose dextrous manipulator," in Proceedings of the IEEE International Conference on Robotics and Automation, Detroit, MI, 1999.

[44] W.-H. Zhu, J.-C. Piedbœuf, and Y. Gonthier, "Emulation of a space robot using a manipulator on ground," in Proceedings of the IEEE International Conference on Robotics and Automation, Washington DC, VA, 2002. 